\title{
An Inquiry-led Personalised Navigation System (IPNS) using multi- dimensional linkbases
}

\author{
Panchit Longpradit ${ }^{\mathrm{a}}$, Wendy Hall ${ }^{\mathrm{b}}$, Robert J Walters ${ }^{\mathrm{b}}$, Lester Gilbert ${ }^{\mathrm{b}}$, Quintin Gee ${ }^{\mathrm{b}}$, Gary \\ B Wills ${ }^{b^{*}}$ \\ ${ }^{a}$ School of Information Technology, Phetchaburi Rajabhat University, Thailand \\ plongpradit@scaat.th.edu \\ ${ }^{\mathrm{b}}$ School of Electronics and Computer Science, University of Southampton, UK \\ \{wh, rjw1, lg3, qg2, gbw\}@ecs.soton.ac.uk
}

\begin{abstract}
The simplicity of the hypertext model behind the World Wide Web is a factor in its success, but this simplicity brings limitations. One of these limitations is embedding links in documents. Open Hypermedia addresses this by instead storing them in separate link databases. Meanwhile, the Adaptive Hypermedia approach seeks to enhance a user's experience by inserting personalised additional content and links on the web page. However, these techniques do not offer the user any control over the adaptation. In this paper, we propose the concept of a multi-dimensional linkbase for adaptive links presentation. Links are created and stored in a single, multi-dimensional, linkbase that provides presentation links based on the user's preferences and profile. We present a web-based system (IPNS) that implements this multi-dimensional concept for controlling its personalisation of hyperlinks. We give the results of our evaluation, which confirm that user-controlled adaptation is a satisfactory approach to providing users with control over personalization, and can alleviate the link overload problem.
\end{abstract}

Keywords: Multi-dimensional linkbase, Adaptive hypermedia, Open hypermedia, Link augmentation, FOHM, Auld Linky

\section{Introduction}

Tim Berners-Lee incorporated the idea of hypertext within the Internet to produce the World Wide Web, the advent of which has changed how people develop and distribute information today. The web now hosts billions of pages of information. The simplicity of the hypertext model behind the web is a factor in its success [3]. Although the web provides users with navigational functionality using hypermedia links and search engines, some shortcomings of the current web model as a hypermedia application is that links are hard- 
coded and embedded within documents, and there is no support for associative linking [16]. These issues can be seen in the problem of updating and maintaining web materials, and in the difficulty of finding the right information.

Hypermedia is a concept that allows authors to structure information in a non-sequential network of different forms of media. Information in a hypermedia system can be presented in any textual or pictorial form, and the information is inter-related by means of linking and indexing $[34,38]$. In this environment, users have freedom to browse and navigate the information space using links presented to them. Traditional hypermedia systems suffer from usability problems, such as cognitive overload and "lost in hyperspace" [11], that arise due to the excess of information and multiple navigational paths. Many "hyperdocuments" are so cross-referenced that users can lose their location and direction.

The Open Hypermedia $(\mathrm{OH})$ community views hypermedia systems as ones that provide services for the integration of information and processes in a distributed heterogeneous environment where there is no distinction between a reader and an author [13]. Traditionally, $\mathrm{OH}$ techniques have been used to provide simple adaptation by inserting additional links on the body of a document from external databases.

Adaptive hypermedia ( $\mathrm{AH})$ is a sub-discipline of hypertext research. AH systems use strategies and frameworks to model their links and provide users with the adaptation of contents at runtime [6].

In this paper, we show how $\mathrm{OH}$ and $\mathrm{AH}$ techniques can be combined to enhance a webbased e-learning system. We introduce the term multi-dimensional linkbase (MDL) to describe a database containing links annotated with different contextual metadata, and we present a prototype system called the Inquiry-led Personalised Navigation System (IPNS), built around the MDL concept.

Section 2 of the paper gives an overview of the research background in $\mathrm{OH}$ and $\mathrm{AH}$. Section 3 introduces the concept of a multi-dimensional linkbase and its characteristics. Section 4 describes the prototype developed, an inquiry-led personalised navigation system. We then discuss the evaluation approach to the IPNS in Section 5, and finally draw conclusions and present potential future work in Section 6.

\section{Background}

Our work on Open Adaptive Links Presentation is centred on two threads in hypermedia research: open hypermedia and adaptive hypermedia. In this section, we give a brief description of these two fields.

\subsection{Open Hypermedia}

OH research originated in the late 1980s [44]. The underlying principle is that links should be separated from the body of a hypermedia document and stored independently in a link database (linkbase). A linkbase can be viewed as a collection of link structures, holding information about the source, the destination and the type of a given link. This approach is implemented using a link service, which acts as a query interface to provide link functionality between an application and one or more linkbases. A link service functions on demand, permitting a client application using it to insert additional links on web pages dynamically from a variety of linkbases. The process of inserting additional links dynamically into an opening web page is referred to as link augmentation [4]. 
It is claimed that the main advantage of the link service approach is that links can be created, added, or modified without changing the original document. Since the links are often related to keywords in the document, even if the text is modified or rearranged, the original links continue to function [4], as long as the keywords are not removed. This approach greatly reduces the information maintenance workload and increases authoring capability [8].

$\mathrm{OH}$ research has grown out of work on the construction of hypermedia across different types of media [28], the provision of conventional link functionality [26], the creation of hypertext across heterogeneous environments [1], and work on distributed hypermedia [2].

At the University of Southampton, OH research commenced in the early 1990s with the creation of Microcosm [13], in which users were provided with dynamic and crossapplication hyperlinks. The Distributed Link Service (DLS) [9] was developed to widen the Microcosm philosophy by supporting multiple users operating in a distributed environment, and to incorporate the web model. At the core of the DLS is a proxy web-server, which manipulates all requests for web pages and returns pages augmented with additional links. The DLS proxy server and an agent system have been integrated to provide contextual linking [15]. COHSE [10] integrates the DLS with ontological services to provide linking based on concepts.

The Fundamental Open Hypermedia Model (FOHM) [22] is an open hypermedia model that uses contextual structures to describe the structure of hypertext objects and the associations between data objects. Auld Linky [24] is a link server designed to store and serve contextual FOHM structures. Auld Linky can be used to respond to users' requests for link matching dynamically, and to provide flexibility in modelling hypermedia structures. FOHM was designed to provide a common language for expressing navigational, spatial and taxonomic hypertext systems.

\subsection{Adaptive Hypermedia}

$\mathrm{AH}$ is an area of research that attempts to increase the functionality of hypermedia applications. The intention is to reduce the cognitive overload and disorientation problems by assisting users with their navigation. Using adaptation techniques [6], a static page can be personalised to meet an individual's needs. Users with different interests and background knowledge are presented with different portions of the same information, or with different information, in the form of content and navigational links. Many systems and frameworks for AH systems and applications have been proposed, such as [3], [7], [14], [17], [20], [23], [27], and [33].

AH covers issues such as shareability, reusability and the Semantic Web [14]. This is still an active area of research, especially in identifying how to provide personalisation and adaptation in web-based learning systems.

Millard et al. suggest that $\mathrm{OH}$ is centred on the design and authoring of hypertext structures (e.g. navigational links) and is less concerned with the way in which information can be personalised and adapted although the 'free-form approach' of $\mathrm{OH}$ can allow for adaptation [23]. The separation of links and hyperdocuments enables additional hyperstructure or links from linkbases to be incorporated at run time. This enables existing web pages to be adapted with additional links based on the user's selection.

On the other hand, AH concentrates on the way in which information can be personalised and adapted at content or link-level, based on a user's profile or preferences. The adaptation process takes place dynamically, based on a user's status at run time, but the process is predefined at design time by the hypertext author. 
There have been attempts to reconcile the two fields and bring the $\mathrm{OH}$ architecture and link models to the field of AH ([5], [17]). For example, one approach used an $\mathrm{OH}$ contextual structure to explain and clarify Brusilovsky’s adaptation taxonomy ([3], [5]).

\subsection{Research problems}

Despite its advantages, there are problems with traditional $\mathrm{OH}$ link augmentation techniques. The main difficulty is that link augmentation is based on replacing specified or visited keywords or phrases, which leads to the problems of link overload such as:

- Inserting too many links in an existing web document (prolific linking [10]), where every keyword becomes a link [4]. These links might be irrelevant or out of place when they fail to support the document's context [15].

- Deciding which link to favour where multiple destination links are associated with the same anchor keyword.

In addition, systems with link augmentation commonly provide a linkbase. They may also provide a set of linkbases (multiple linkbases), based on representing a conceptually similar rationale as an individual linkbase [12]. Multiple linkbases could serve our purpose until a link can be a member of more than one group, when the separation of linkbases is impossible. For example, a link that is for beginners can be placed in a beginner linkbase, one for experts placed in an expert linkbase, and a link for students in a student linkbase. However, with the current approach, a link for beginner students has no linkbase in which to reside.

A sub-dimension within a dimension cannot be modelled with separate linkbases. For instance, suppose an expertise linkbase has a sub-dimension 'level of competency' with values: elementary, trainee, ..., proficient. The multiple linkbase approach does not allow a link structure to be modelled more than once.

One of the criticisms of adaptive systems are that users do not always understand, or find it difficult to understand, or do not have any influence over, the way the system adapts the contents and links [27]. AH research has taken users' differences in background, tasks, and interests, into consideration when providing enhanced usability of hypertext functionality. $\mathrm{AH}$ is less concerned with information structure and system architecture, and more focused on applying its strategies to content and links presented to users, based on the user's profile [23]. AH allows the same, or different, information to be presented in a number of dissimilar ways. The early AH systems and applications were centred on the employment of adaptive techniques. AH researchers argue that the technique does not prohibit but instead recommends the navigational paths users could choose.

However, the fact that different information from the same page is presented to different individuals, is likely to create the 'difference' problem. For example, two users accessing an adaptive system will probably observe differences in what they see. If one prefers the other's version, they cannot do anything about it because they do not have control over the adaptation, which arises from having different user profiles. Another example is the adaptive system that detects a change in a user's interest, and adapts the contents and links to match. Although the system might detect a shift of interest, the user is actually changing his or her context. The problem arises from the users seeing the automatic change in content, and then becoming confused as to whether or not to follow the change.

It is hypothesised that a personalised system, which allows users to interact with the adaptation system, might provide a sound basis for improving the navigation process and this is one of the primary objectives of this work. 


\section{Multi-dimensional linkbase}

A multi-dimensional linkbase (MDL) is a concept that describes a single linkbase containing sets of links annotated with metadata, so that these links appear to exist in many different contextual dimensions at once. For example, these links might signify dissimilar dimensions of expertise, and provide the contextual structure that enables and disables their visibility. The domain could comprise $\mathrm{N}$ expertise dimensions and each expertise dimension could be classified into M sub-dimensions. Users who have different dimensions of expertise, and possess dissimilar levels of expertise within each dimension, would be provided with diverse representations of links from each dimension of expertise. This takes into account the fact that users with different levels of expertise require additional information differently when navigating. The user should only view presentations of links appropriate to their level and these links should only come from the user's chosen dimensions of expertise. For example, a skilled English historian with no expertise in Asian history will require different links presented whilst browsing text on Asian history, compared with another user who is an Asian historian.

Incorporating the MDL concept with a link server (acting as a query interface and supplying links on demand) enables links to be conditionally presented and personalised to the user, based on their individual profile. That is, the link server inserts supplementary links from the MDL on a web page dynamically in relation to a user's expertise dimensions and levels of expertise in each dimension.

The MDL is more efficient than using multiple linkbases, where each linkbase represents one dimension of context to store link structures. In addition, when the MDL is implemented for supporting link adaptation, MDLs retain the simplicity of adaptation, allowing users to see the working behaviour of the adaptive system and allowing them to configure the link personalisation to suit their needs. One of the benefits of the MDL is alleviating the problems of prolific linking and out-of-place links, while maintaining the user's understanding of the adaptation process.

Multi-dimensional database structures have advantages over relational databases since "it is more efficient to represent the dataset with a multi-dimensional array rather than a relational table as it reduces the duplication in the relational table, increases performance and provides ease of maintenance" [30].

In addition, the MDL concept is aimed at making possible those situations where multiple linkbases are impractical. For example, a link can be annotated as a member of more than one group. That is, not only can a link in the MDL be designed for beginners, but that same link can also be defined to be visible for experts. Moreover, it is possible that a link in the MDL can have a sub-dimension. Thus, links can be supplied for a user for beginners (in expertise dimension), for English speakers (in language dimension), and favouring audio presentation (in media dimension).

\section{$4 \quad$ A prototype system}

The MDL concept has been implemented in our web-based system, the Inquiry-led Personalised Navigation System (IPNS). We defined our implementation as: $a$ system that allows users not only to browse the constructed materials at their own pace, but also to search for information in a goal-directed fashion using the tools provided. The term Inquiryled was chosen in particular to denote the inquiring action and to emphasise that these inquiry tools were implemented as 'add-ons', by which we mean that the tools can be 
functional on demand, dependent on the user's preference. These tools enable users to pursue more exploratory navigational strategies.

An inquiry-led navigation system, based on the definition given above, possesses the following characteristics.

- It offers active/self-driven navigation by involving users in the process of making navigational decisions.

- It motivates users with inquiry tools aimed at assisting the user's navigation.

- It provides some degree of user control.

- Its tools can serve as scaffolds to support users in the inquiry process.

- It values the experience and knowledge that users possess and bring to the navigational process, whilst at the same time allowing them to deepen their inquiry.

There are a number of reasons why inquiry-led design was chosen to provide navigation in our web-based IPNS prototype.

- It promotes participation, by encouraging users to become actively involved in the process of navigation.

- It offers users sophisticated control of link presentation and personalisation. (A major criticism of adaptive systems is that users do not have such control.)

- It allows the user to explore the materials, and to inquire about information using the tools provided.

- It allows the user to experiment with the functionality at runtime, and observe and control the result.

- It allows the user to set the personalisation of links at any time to their preference and expertise, with changes taking effect immediately.

Since users can see the working behaviour of the system, and make the adaptation work better for them, we believe this will alleviate the 'too-many-irrelevant-links' problem.

\subsection{Conceptual design}

Figure 1 demonstrates a conceptual design of the IPNS, while Figure 2 shows the IPNS architecture, and how each operational module is related to, and communicates with, the other components. The IPNS comprises four models, required in any AH system.

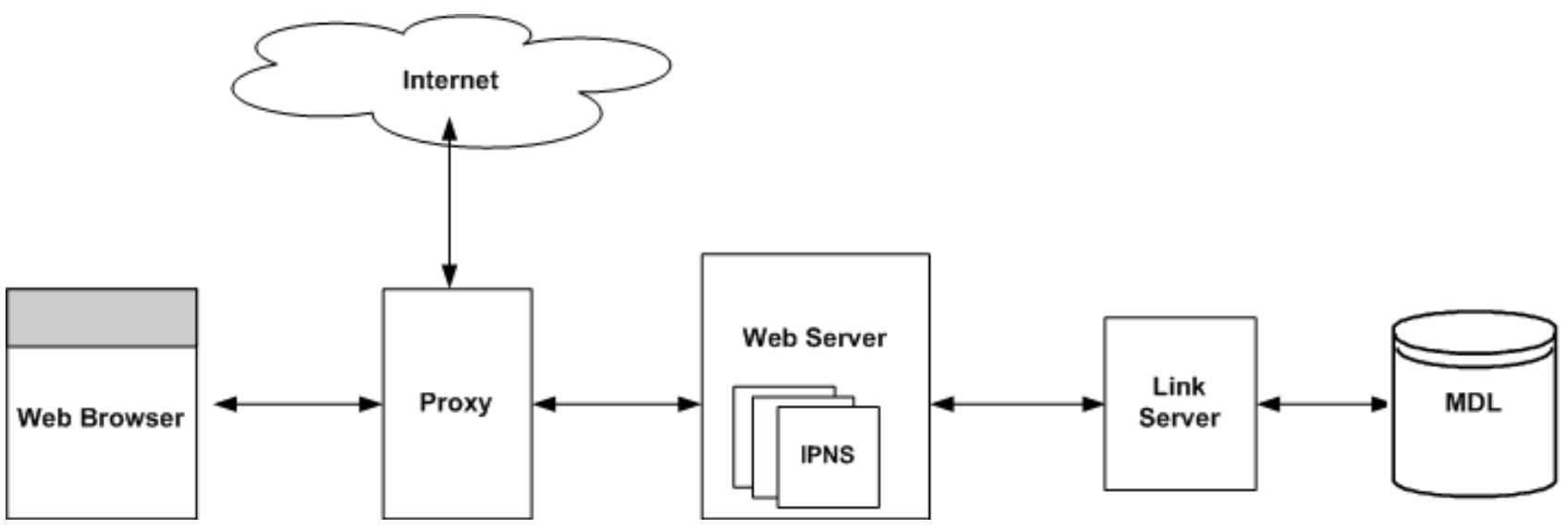

Figure 1. A conceptual design of IPNS 


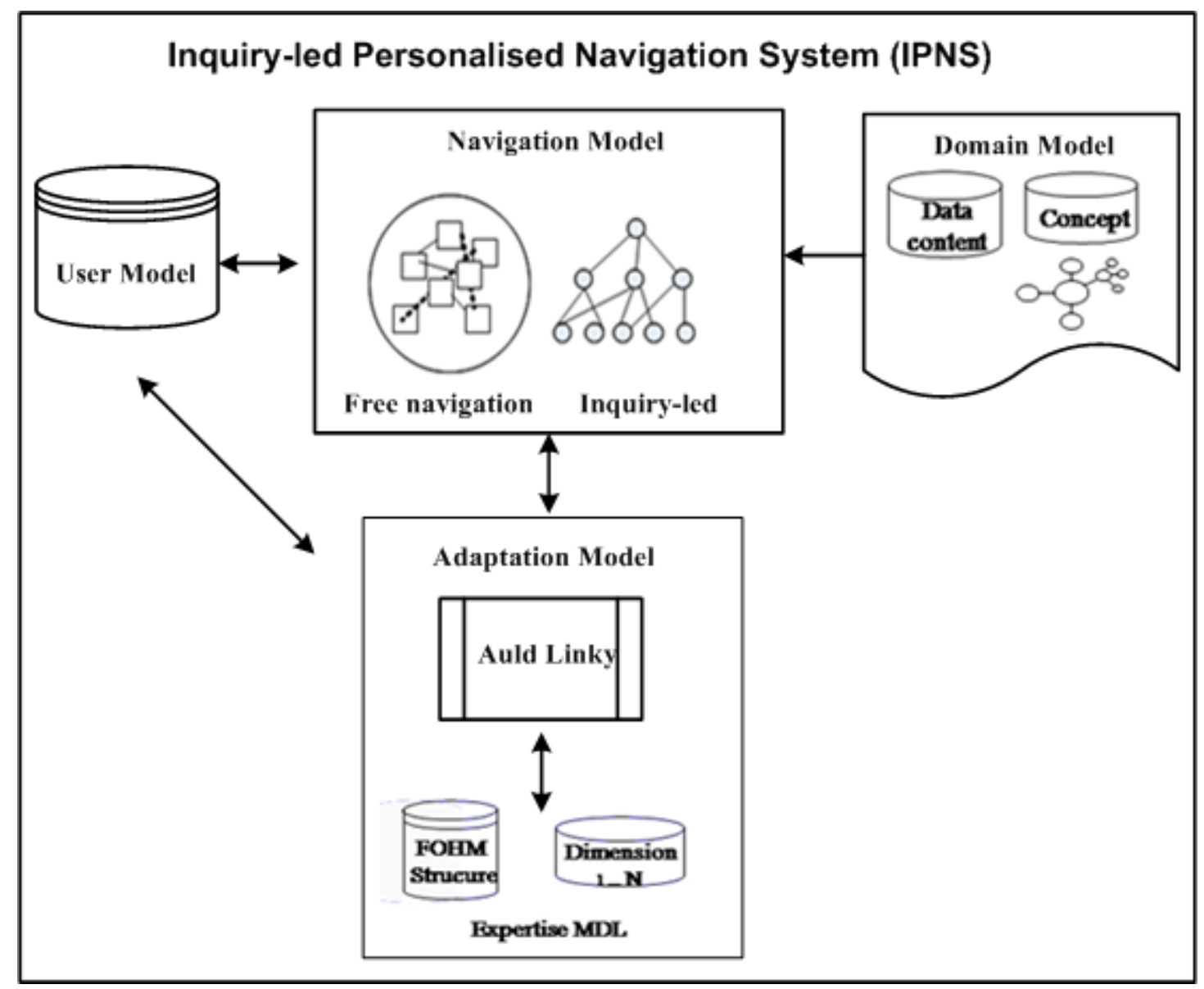

Figure 2. IPNS architecture

- Domain model represents how data content in IPNS is organised to support its navigational module, E-book, and concept relationships (or specifically defined 'ontology'). To facilitate inquiry-led navigation, the domain was modelled as a concept hierarchy with additional ontological relationships between concepts. The subject domain was divided into smaller topics and concepts, where each concept was related to another concept or data item by means of a FOHM structure with one of a set of established relationship types. Users can access this domain ontology through an inquiry interface.

- Navigation model concerns the way users are enabled to navigate the information space in IPNS. Communication between the domain model and the navigational model occurs in two ways: free navigation (browsing through the electronic textbook), and inquiry-led (navigating with the aid of inquiry tools). The first method allows users to browse the material in an informal way at their own pace. The links for free navigation are those embedded in the text, and the structural navigation is provided by the website. Inquiry-led navigation begins when the user selects one of the inquiry tools provided to support their process of navigation. These optional tools were designed to assist the user with more navigational strategy, for instance, by providing a means to personalise the presentation of links, finding what topics they are looking for, and looking for more links to related topics.

- Adaptation model deals with the navigational interfaces, link augmentation and the personalisation of links. The adaptation model is the component that draws the navigational and user models together. Its three components are: personalised links 
assistant module, inquiry links module, and the 'follow links' module. Personalised Links module concerns both the presentation and the personalisation of links from the MDL. The Inquiry Links interface concerns the presentation of links, based on the defined ontology. The Follow Links module is centred on links presentation, based on the Microcosm [29] philosophy, which allows users to look for links that follow a particular topic. Auld Linky provides appropriate views on the linkbases (Expertise MDL, Inquiry Linkbase, and Glossary Linkbase) using its culling process, and returns the relevant links matching a given context.

- User model contains information about the users, including username, password, and levels of expertise in the various skill dimensions. It also establishes the personalisation settings for an individual user. The IPNS initially stores a record of the user's general information, following which the user will be asked to select their initial adaptation. This selection provides preliminary values to be assigned to the model of that user, which will then be used for the personalisation of their Expertise $M D L$ links. Whenever they want, users are allowed to amend their levels of expertise, resulting in the user model being dynamically modified. Not only does the amendment of these expertise values take effect immediately, but it also results in revised augmentation on the page.

\subsection{Expertise MDL}

In our implementation, we chose the domain of Food Science and Technology. We purposely selected a topic that had little to do with IT, yet would still be a science subject. Baking is a universal subject and of interest to many people. With the addition of science and technology, it offers a rich and systematic domain. Baking technology can be viewed as a food production operation, which concerns raw materials (input), food transformation processes (mixing and baking), and products (bakery products). This approach enabled us to apply a model for the classification of links, and allowed us to identify skills within the subject domain as different dimensions of expertise, for example, expertise about raw materials, operations, products, and food hygiene. The resulting Expertise MDL comprised links that related a keyword to its additional elaboration or explanation. The MDL encompasses three example dimensions of expertise: Subject expertise, Language expertise, and Assessment preference. In principle, there can be any number of dimensions.

- The Subject dimension was classified into three sub-dimensions: raw materials (the input to the operations, i.e. basic and advanced science, bakery ingredients, and equipment), bakery operations (issues relating to mixing and baking technologies), and bakery products (the output of the transformation process, i.e. bakery products and hygiene). These subject links can be visually enabled or disabled with the following four options - 'no link', 'basic', 'advanced' and 'all links' - for the user to make a selection. The 'no link' option assumes that the user has a sophisticated degree of knowledge of the subject domain and therefore does not require assistance from additional links. 'Basic' presents the user with links relating to additional information about the basic concept. The 'advanced' option offers links describing more advanced information about the subject domain. Finally, 'all links' generates all subject expertise links available on the page.

- The Language dimension allows the user to see a chosen keyword augmented with a selection of languages, such as Latin and Spanish as well as English. 
- The Assessment preference dimension allows users to select the interactive and non-interactive versions of the assessment test.

The navigational links in the IPNS application are all held in the Expertise MDL. If no links are chosen using the tool provided, users will only see the traditional static structural links for navigating between pages.

\subsection{Link structures}

The Expertise MDL is expressed as FOHM structures. The essential components of a FOHM structure [22], as shown in Figure 3, are as follows.

- Associations represent the relationships between Data Items and other Associations.

- Bindings specify the connection between Associations and Data Items.

- References are pointers to the entirety of Data Items, or parts of the Data Items.

- Data Items represent pieces of information that can be words, paragraphs, concepts, or entire documents.

- Context objects can attach to any part of the link structure, and describe conditions of the visibility of objects, particularly the Data Items.

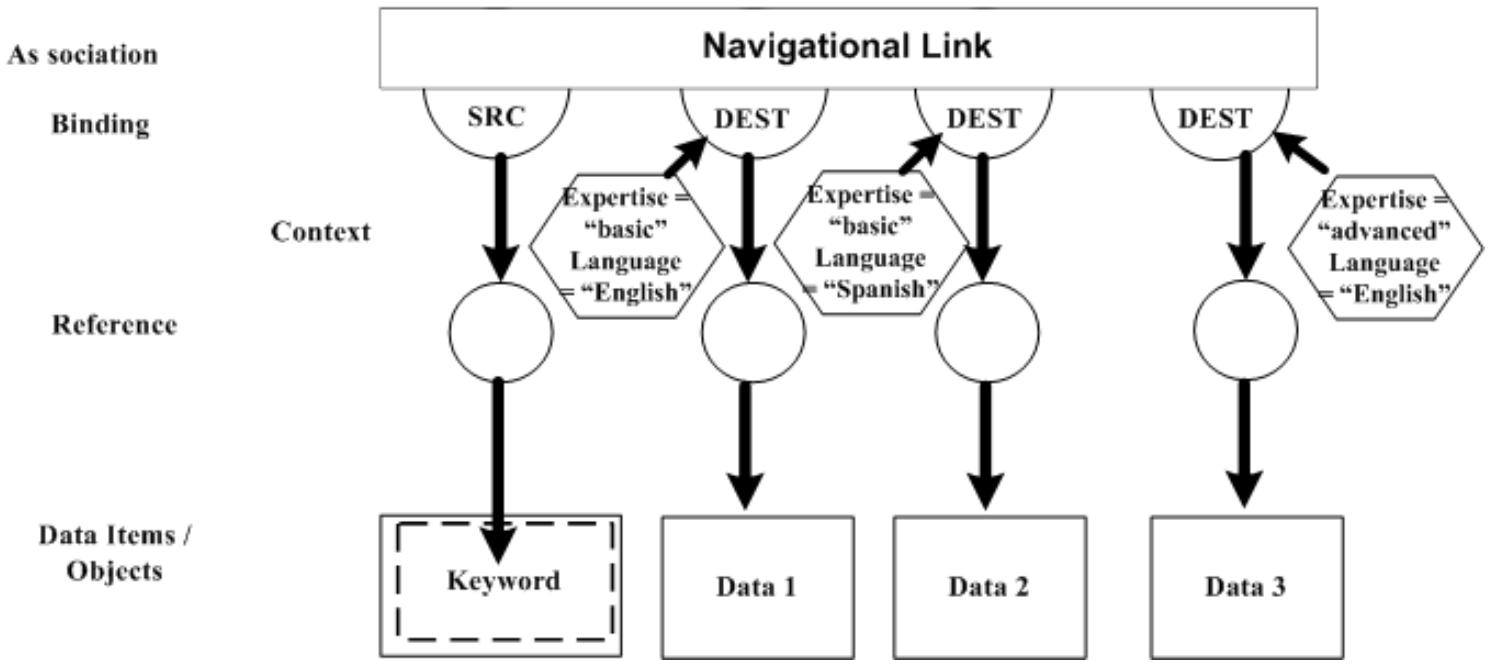

Figure 3. FOHM link structure

FOHM differs from other $\mathrm{OH}$ models in that it has contextual structures that can be used to describe the metadata about the hypertext structures [22]. A FOHM Context and Behaviour object can be attached to any part of the hyperstructure, and at several different points in the hyperstructure. This context attachment provides a personalisation mechanism through defining the conditions of visibility. For example, one link can be made visible only to advanced users, whereas another link can be specifically displayed to, and only accessible by, beginner users. This work has used FOHM and Auld Linky as a means to implement IPNS and expanded some of the FOHM capability. While FOHM allows for many dimensions of context, the concept of $\mathrm{N}$-dimensions of context as an $\mathrm{N}$-dimensional linkbase has not previously been implemented in FOHM, and neither have user profiles. Therefore, we have enhanced the context mechanism in FOHM to facilitate the personalisation and adaptation based on an individual user.

In addition, we extended FOHM to provide semantic representation of concepts or associations of the subject domain as FOHM-based structures. These concept structures relate 
to other concepts by means of FOHM links with a set of ontological relationship types. The users are then able to access this representation of concepts through the user interface. This use of taxonomy-based ontology aids users in the process of querying a concept and its associated concepts.

A single MDL contains the source and destination information for all links in its group. Individual links within the MDL can have one or many sources and/or destinations depending upon the concept(s) they are representing or associating.

\subsection{Link presentation}

Links from within the MDL are presented to a user, based on the direct mapping between expertise dimensions and their contextual links in the MDL. That is, the user will be provided with the contextual links from the MDL in relation to the user's expertise dimensions. Figure 4 shows the presentation of links in the MDL.

Expertise 1,2,3...N

Expertise Dimension

Language Dimension

N Dimension
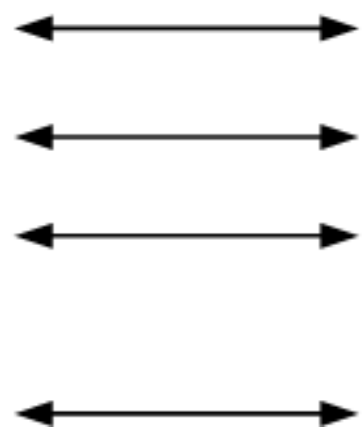

MDL

Expertise links in MDL

Language links in MDL

$\cdot$

N Dimension links in MDL

Figure 4. Presentation of Links with MDL

Our IPNS has been implemented using web technologies to allow the generation of dynamic web contents and to facilitate session tracking. This provides the mechanism for identifying individual users via HTTP responses and requests. The link annotation and augmentation processes are controlled from a proxy server that intercepts user requests and queries the link server for appropriate views of the linkbases. When a browser requests a page, the request is forwarded to the proxy, which in turn communicates with the link server. The link server then finds the links for the current web page, given the context sent by the proxy (as a set of keywords). Links are visible when they match the requested expertise dimension and its level. The link server uses its culling process to return a particular view of the relevant links, which are then returned to the proxy. The proxy parses the FOHM link structure and replaces the textual form of links (from the linkbase) with the anchor tags. The opening web page would then be augmented with additional links.

All link presentation in IPNS is based on the link augmentation technique, i.e. inserting additional links dynamically on existing web pages. Links from the Expertise MDL are shown in dark pink to differentiate them from the traditional static links provided in the domain. Users can make the Expertise links (from the MDL) visible or invisible for each of the expertise dimensions. Figure 5 depicts the view of the interface and the augmented links. In Figure 5 the cake and cake baking links are circled. The links result from the user selecting the Subject dimension - Bakery product (for cake) and Bakery operations (for cake baking) with a sub-dimension of Basic links, as well as selecting the language dimension as English. If the user selects different dimensions, a different set of link will be displayed. For instance, if the user changes their settings for the sub-dimension to Advanced links, the result would then be that the keyword 'cake' and 'cake baking' are no longer links. 


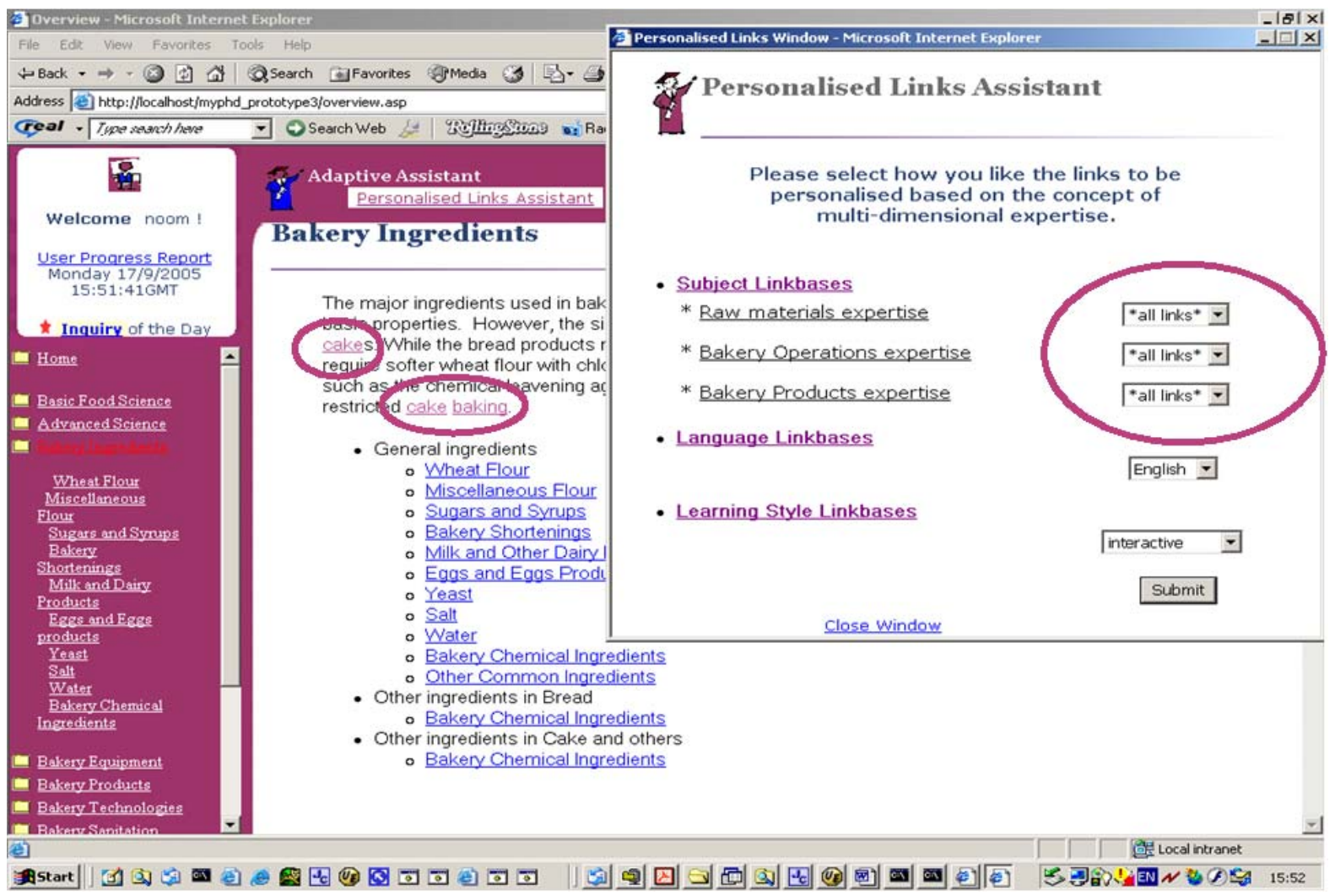

Figure 5. The personalised link interface

\section{Evaluation approach}

Generally, HCI evaluation methods and techniques can be used to assess the usability of hypermedia systems and applications. Interviews, questionnaires, session logging, and observation have been suggested [41], to name just a few. In addition, the cognitive walkthrough method can be applied, with the emphasis on all possible routes the user can take while interacting with the system [39]. However, peculiar to hypertext is the problem that users subjectively navigate through the information space, (such as: where we are now? and where we can go next? [36]), as is the problem of keeping track of pages they visit [40]. This complexity induces the cognitive overload problem, and results in a need for different evaluation criteria [43]. Hothi [36] reported that the usability of a hypertext application relies not only on the user-friendliness of the interface, but also on a combination of issues such as the hypermedia system engine (presentation and navigational support) and the contents and structure of the information space.

Software metrics are other evaluation criteria addressed by a number of researchers. Babiker et al. [31] proposed a metric for evaluating hypertext systems usability based on three issues:

- access and navigation (how easy it is for users to navigate within hypertext documents)

- $\quad$ orientation (how well users know where they are and where they have visited)

- user interaction (how simply the user can interact with the hypertext system)

which they claimed could provide an effective means of identifying problems associated with the system and also as a basis for comparison between different hypertext systems. The main 
difference between evaluation of interfaces, hypermedia, and adaptive hypermedia lies in the nature of their complexity [36], in particular, the comparisons of those systems with and without adaptivity, and the measurements of the levels of adaptivity provided [35].

IPNS originated from both open hypermedia and adaptive hypermedia. Weinreich et al. [42] pointed out that the availability of many link types is only helpful if the user can distinguish between them. The main emphasis of the evaluation approach to IPNS was therefore to find evidence to support the claim that user adaptation allowed users to have control over personalisation by enabling them to see the working behaviour of the adaptive system. Hence, user control over personalisation (or user-controlled adaptation) formed an underlying principle for evaluation of IPNS.

The evaluation was separated into two rationales. The first goal was to measure the applicability of the user-controlled adaptation provided by IPNS; the second, to find out the usefulness of the user-controlled adaptation provided by the prototype. The results of the evaluation have provided some evidence for the following hypotheses:

- The user-controlled adaptation provided by the MDL concept is applicable

- The user-controlled adaptation provided by the MDL concept is useful for users

Based on these rationales, the evaluation was conducted in two different experiments: Heuristic and Empirical.

\subsection{Heuristic Evaluation}

Heuristic evaluation may be employed to provide the criteria or 'heuristics' for identifying some existing problems in the way a concept is applied (but not to verify the non-existence of problems), and to offer a quick result with low cost. We used the nine usability heuristics suggested by Nielsen [25]: Flexibility and Efficiency of Use; Easy to Comprehend; Easy to Remember; Pleasant to Use; User Control and Freedom; Few Errors; Consistency; Aesthetic and Minimalist Design; and Match between the System and the Real World. In addition, the purpose of this heuristic evaluation was to report on the strengths, weaknesses, and applicability of the user-adaptation approach provided by the IPNS rather than assessing usability problems in general.

Expert evaluators were individually asked to read a written introduction to the research objectives, the MDL concept, and the IPNS prototype (see Appendix A). Once they completed the introduction, the evaluators were presented with the prototype and given the opportunity to become familiar with it and its tools. Then, the evaluators examined the prototype by a 'walk through', and scored it against the heuristics using a 5-point Likert scale. The evaluators were asked to identify good and bad aspects of the prototype, and to comment on potential improvements. At the end of the session, the evaluators formed an informal focus group to reflect on what they had found, where they agreed and disagreed, and how they thought they would react if other ways of applying the concept to the system were implemented.

The heuristic evaluation was performed by a set of nine independent expert evaluators who individually carried out tasks on IPNS and critically judged the prototype against the heuristics, indicating their responses on the form provided. The nine expert reviewers consisted of six computer science postgraduate students, two computer science researchers, and one IT related professional, comprising 4 males and 5 females.

Figure 6 shows the results of the heuristic evaluation. This demonstrates that there were no major differences amongst the nine experts. For most heuristics, the responses elicited ranged 
between strongly agree and neutral. None of the evaluators strongly disagreed with any of the nine heuristics. This indicated that the user-adaptation approach provided by the MDL concept did conform to its requirements and heuristics. For instance, all evaluators agreed or strongly agreed with the heuristic 'Flexibility and Efficiency of Use', that is, the IPNS was able to deliver its functionality (i.e. the presented links were rightly functional and acceptable both to experienced and inexperienced users, and allowed users to perform their task). Most evaluators agreed that the IPNS did enable the user to choose the system functions (i.e. link presentation and personalisation) and allowed the users to have control and freedom in interacting with the system (the heuristic 'User control and freedom'). Similarly, the results from the evaluators indicated that the IPNS prototype provided a modest design and did not contain irrelevant information (the heuristic 'Aesthetic and minimalist design').

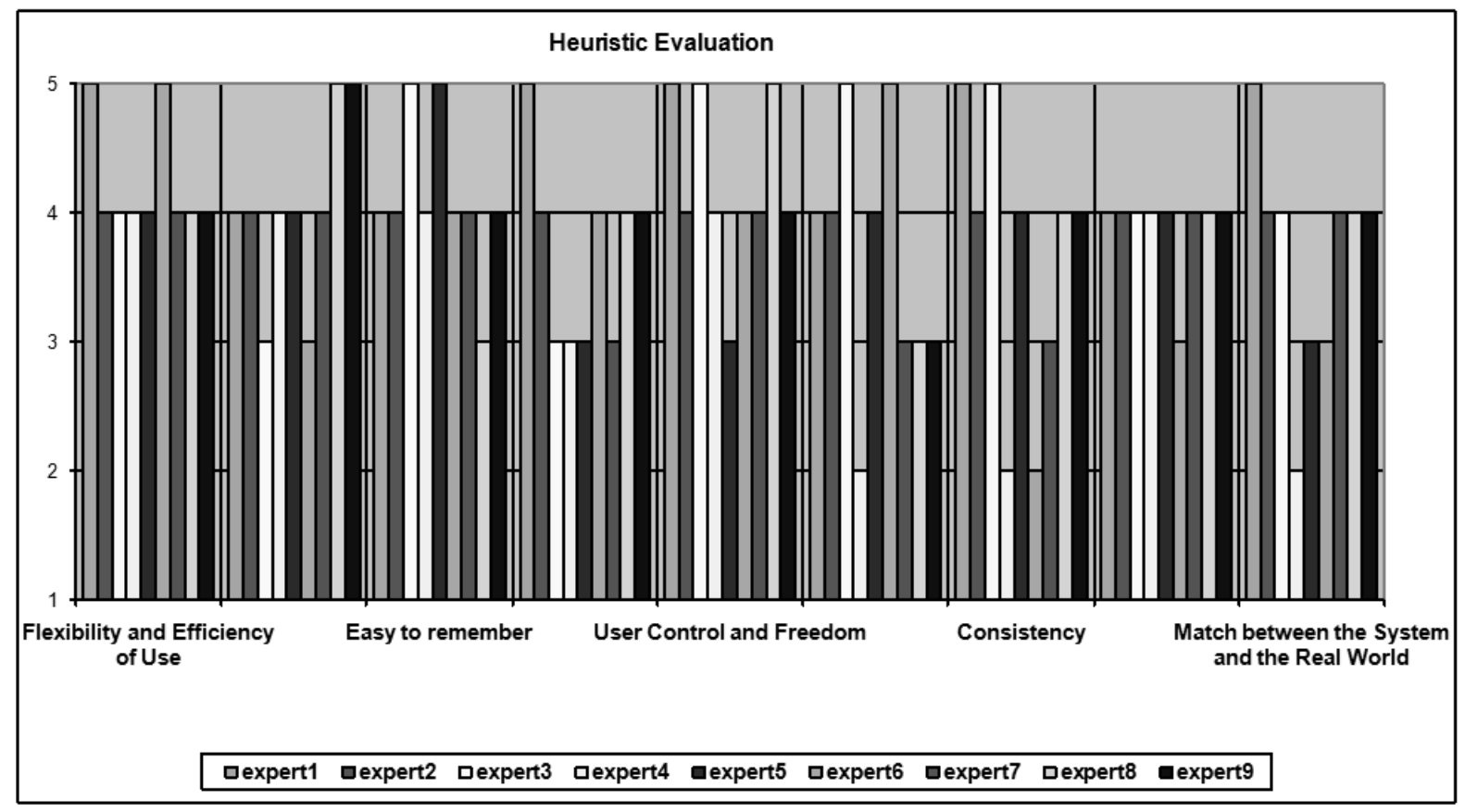

Figure 6. The overall result of the heuristic evaluation

The majority of the free text comments related to improvements in the IPNS interface and not the MDL concept. The majority of the comments on the MDL concepts were positive, for instance:

- "The system provided more functionality and the tools were user-friendly. They reacted immediately with input.”

- "Appearance and disappearance of links definitely satisfy users."

- "It was good that the system allowed users to select which dimensions of links to appear and it saved time for users to be able to get rid of irrelevant contents (links)."

The expert reviewers also suggested ways that the implementation could be improved:

- "The design of the assistant tools could be improved to make them more usable. For instance, it would be useful to know which linkbase the links were appearing from, or which dimension the word came from, e.g. a different colour per linkbase.”

- "An issue that users could take too much time to configure the setting that they want, needs to be taken into consideration. This is because it could cause users to discontinue using the system and the tools provided." 


\subsection{Empirical Evaluation}

Empirical evaluation was designed to find out whether the user adaptation provided by the IPNS was useful and meaningful to users. This evaluation was based on usability criteria in ISO/DIS 9241-11 [18]. In our case, these can be resolved as:

\section{Effectiveness}

Efficiency

User Satisfaction solve prolific linking or irrelevant links, provide ease of navigation, make sense to users, provide appropriate user interface

maximise user control and freedom, speed of navigation, percentage of task completed, and efficiency of use user's opinion of the system, whether the user likes interacting with the prototype, whether the user prefers the IPNS

For this evaluation, there were 24 subjects comprising 8 males and 16 females. Six had a computer science background; the remaining 18 were from a non-computer science background, while their ages ranged from 23-41. The evaluators were randomly assigned to two equally sized groups, such that each group started with a different system in order to control for learning effects. The three systems used were:

- System A non-personalised system (no link)

- System B with all links augmentation but no control over links presentation

- System C with control over links personalisation and presentation (IPNS)

A summary of the experiments and their purposes is given below.

To investigate the effectiveness of the user-controlled adaptation provided by the IPNS (System C) compared to navigation without the presence of personalised tools (System A). The task for this experiment was to answer nine questions in fifteen minutes. The dependent variable was the percentage of the task completed; this took into account the number of correct answers and the time taken. The questions types were either free text or multiple-choice. Example questions were:

Q1: Find out what carbohydrates are and the category they are in. (free text answer)

Q2: Which of the following describes the meaning of 'gelatinization'?

a) The process where proteins are hydrolysed and broken into several amino acids.

b) The process where starch granules take up water and starts to swell and form gel.

c) The process where bacteria have infected food and caused food poisoning.

d) None of the above

e) I am not sure 
Experiment 2(a) To examine the efficiency of the user adaptation provided by the IPNS (System C) compared to the system without the presence of personalised tools (System B). The task for this experiment was to locate some of the terms in the subject domain, where the evaluators were asked to record their start and finish times. Each task had a time limit of 5 minutes. The percentage of tasks completed (as in task 1) and the speed of navigation of each user was measured, along with the time taken to identify the terms, or a percentage of these if they reached the time limit.

A typical question was 'Find the following keywords: Crumb, Gluten, Caramel Rope.'

Experiment 2(b) To examine whether users prefer the user-controlled adaptation provided by the IPNS (System C) more than non-personalised systems (System A and System B). The purpose of this experiment was to obtain the evaluators' subjective feedback about the systems. Evaluators were requested to answer the questionnaire related to each system qualitatively and to compare the three systems and list the one they preferred.

Experiment 3 To study the user satisfaction of the user-controlled adaptation provided by the IPNS (System C). The aim was to obtain the evaluators' subjective feedback solely about the IPNS prototype in which they were asked to complete the questionnaire using a 5-point Likert scale. The scales used for measuring user satisfaction were based on the Software Usability Measurement Inventory (SUMI) Affect, Control, Efficiency, Helpfulness, and Learnability [37] - and scales for evaluating industrial hypermedia, Navigation and Comprehension [32].

The data was analysed using SPSS version 13, a statistical package for social science research.

\subsection{Summary of the results}

- Experiment 1 showed that the mean percentage of task completed was significantly higher using the set of links presented by the IPNS prototype compared to navigation without the presence of personalised features $(\mathrm{t}=3.329$, $\mathrm{df}=15, \mathrm{p}<0.05)$.

- Experiment 2(a) showed no significant difference in the mean percentage of navigation completed using the set of links presented by the IPNS prototype compared to navigation without the presence of personalised features.

- Experiment 2(b) showed that mean user preference rating for the user-controlled adaptation provided by the IPNS was significantly higher than for non-personalised systems. It also demonstrated that the IPNS was useful as it allowed the selection of links to be displayed based on users' preferences. In addition, the mean user rating of control over link presentation and personalisation was significantly higher in the IPNS system than in the non-personalised systems.

- Experiment 3 showed statistically significant differences in mean user ratings of their interactions with the system. These results are summarised in Table 1, showing that users liked the adaptation provided by the IPNS which allowed them to have control 
over links presentation and personalisation, and that this control was useful, helpful, and easy to understand.

\begin{tabular}{|c|c|c|c|c|c|}
\hline \multirow[b]{2}{*}{ Scales } & \multicolumn{4}{|c|}{ Descriptive Statistics } & \multirow{2}{*}{$\begin{array}{c}\text { One-sample t-test } \\
\text { (Difference from 0) }\end{array}$} \\
\hline & $\mathrm{N}$ & Mean & $\begin{array}{c}\text { Std. } \\
\text { Deviation }\end{array}$ & $\begin{array}{l}\text { Std. Error } \\
\text { Mean }\end{array}$ & \\
\hline Affect & 24 & 3.71 & 2.255 & 0.460 & $\mathrm{t}=8.056, \mathrm{p}<0.01^{*}$ \\
\hline Control & 24 & 3.29 & 1.876 & 0.383 & $\mathrm{t}=8.595, \mathrm{p}<0.01 *$ \\
\hline Efficiency & 24 & 3.75 & 2.132 & 0.435 & $\mathrm{t}=8.619, \mathrm{p}<0.01^{*}$ \\
\hline Helpfulness & 24 & 3.13 & 2.290 & 0.467 & $\mathrm{t}=6.685, \mathrm{p}<0.01^{*}$ \\
\hline Learnability & 24 & 3.92 & 2.535 & 0.518 & $\mathrm{t}=7.568, \mathrm{p}<0.01^{*}$ \\
\hline Navigation & 24 & 3.63 & 1.689 & 0.345 & $\mathrm{t}=10.513, \mathrm{p}<0.01^{*}$ \\
\hline Comprehension & 24 & 4.29 & 1.334 & 0.272 & $\mathrm{t}=15.755, \mathrm{p}<0.01^{*}$ \\
\hline \multicolumn{6}{|l|}{ Overall reactions } \\
\hline Satisfactory & 22 & 0.91 & 0.426 & 0.091 & $\mathrm{t}=10.000, \mathrm{p}<0.01^{*}$ \\
\hline Easy & 23 & 1.04 & 0.475 & 0.099 & $\mathrm{t}=10.543, \mathrm{p}<0.01^{*}$ \\
\hline
\end{tabular}

Table 1: Summary of the statistical results for Experiment 3

In general, the results revealed that the MDL concept could support adaptive hypermedia by allowing the users to have more control over personalisation of links in order to make adaptation work better for them, and hence could help the users avoid the link overload problems caused by the open hypermedia's link augmentation technique. The system implementation has established that the MDL concept has presented a different view of representing a linkbase for link personalisation, which resulted in additional functionality to support the process of inserting additional links on the body of a document. The main emphasis of the evaluation study was therefore to show that, by allowing users to have control over personalisation of links, IPNS would give the affordances the user expected from the adaptation. In this context, 'control' is enabling users to see the working behaviour of the system, by means of the direct manipulation of the MDL and other linkbases.

\section{Conclusions}

This paper presents a method for hyperlinks presentation, based on the concept of a multi-dimensional linkbase. Sets of links are created and stored in a single linkbase, and signify multiple dimensions of expertise. Each MDL provides presentation links based on a user's preferences and profile.

The MDL concept represents a method of storing links in a linkbase with additional functionality to support the link augmentation technique - the process of inserting additional links on the body of a web document. It increases the likelihood of users having a clearer and easier understanding of the adaptation process, and facilitates user control over presentation of the links. Hence, it alleviates some link overload problem caused by the traditional $\mathrm{OH}$ technique. 
Implementing the MDL concept for open adaptive links presentation shows the practicability that representing links within MDL makes possible; multiple destinations from the same navigational link. Most systems with link augmentation processes, base their link insertion on replacing individual keywords or phrases in the document, which results in one keyword offering only a single destination. This common practice can result in prolific linking. In contrast, the MDL concept enables the same keyword in the same context to be realised as links pointing to different destinations, dependent on the user-chosen contextual dimensions of expertise in the linkbase. For instance, a keyword 'rice' could become a link pointing to different destinations, such as 'chemical constituents', 'nutritional value', or 'trading market for agricultural products'.

Web-based learning environments, in particular, could gain benefits from the MDL concept. Considering different expertise as different contextual dimensions would allow the learner to be presented with links or contents matched to their levels of expertise. For instance, in an MDL with dimensions Learning Styles (e.g. visual, auditory, or tangible), and Pedagogy (e.g. directive, exploratory), the student could be provided with the contents and links according to their learning style and instructional preference. With the implementation of the rules or inference engine, the system's decision-making can be automatic, based on the result of the individual user model.

Future work will address the integration of different MDLs, and investigate the issues of enhancing and facilitating shareability and reusability.

\section{References}

[1] Anderson, K.M., Taylor, R.N. and Whitehead, E.J. 1994. Chimera: Hypertext for Heterogeneous Software Environments. Proceedings of the ACM European conference on Hypermedia technology ECHT, pp. 94-107.

[2] Andrews, K., Kappe, F. and Maurer, H. 1995. Serving Information to the Web with Hyper-G. Proceedings of the Third International WWW-Conference, Darmstadt, http://www.igd.fhg.de/www/www95/papers/105/hgw3.html.

[3] Bailey, C.P. 2002. An Agent-Based Framework to Support Adaptive Hypermedia, PhD Thesis, School of Electronics and Computer Science, University of Southampton, UK.

[4] Bailey, C., El-Beltagy, S.R. and Hall, W. 2001. Link Augmentation: A Context-Based Approach to Support Adaptive Hypermedia. Proceedings Hypermedia: Openness, Structural Awareness, and Adaptivity. Århus, Denmark, LNCS 2266, 8, pp. 239-251.

[5] Bailey, C., Hall, W., Millard, D.E. and Weal, M.J. 2002. Towards Open Adaptive Hypermedia, Proceedings of the Second International Conference on Adaptive Hypermedia and Adaptive Web Based Systems. LNCS 2347, pp. 36-46.

[6] Brusilovsky, P. 2001. Adaptive Hypermedia. User Modeling and User-Adapted Interaction, 11, pp. 87-110.

[7] Brusilovsky, P. and Pesin, L. 1998. Adaptive navigation support in educational hypermedia: An evaluation of the ISIS-Tutor. Journal of Computing and Information Technology, 6(1), pp. 27-38.

[8] Carr, L., DeRoure, D., Hall, W. and Hill, G. 1995. The Distributed Link Service: a tool for publishers, authors and readers. Proceedings of the Fourth World Wide Web conference, Boston.

[9] L. Carr, D. De Roure, H. Davis, and W. Hall, Implementing an Open Link Service for the World Wide Web. World Wide Web Journal, 1998, 1(2), pp. 21-33. 
[10]Carr, L., Kampa, S., Hall, W., Bechhofer, S., Goble, C. and Horal, B. 2002. Ontological Linking : Motivation and Case study. Proceedings of WWW2002.

[11]Conklin, J. 1987. Hypertext: An Introduction and Survey. Computer, 20(9), pp. 17-41.

[12] Crowder, R.M., Wills, G.B., Heath, I. and Hall, W. 1997. Application of Hypermedia in the Factory Information Environment. Proceedings of Factory 2000, 5th IEE Conference on Factory Automation, pp. 411-415.

[13] Davis, H., Hall, W., Heath, I. and Hill, G. 1993. Towards An Integrated Information Environment With Open Hypermedia Systems. Proceedings of the Fourth ACM Conference on Hypertext, Milan, Italy, pp. 181-190.

[14]De Bra, P., Aroyo, L. and Chepegin, V. 2004. The Next Big Thing: Adaptive Web-based Systems. Journal of Digital Information, 5.

[15]El-Beltagy, S., Hall, W., De Roure, D. and Carr, L. 2001. Linking in Context. Journal of Digital Information, 2(3).

[16]Hall, W. 2000. As We May Learn: The Application of Hypermedia in Education. Computing \& Control Engineering Journal, 11(4), pp. 173-178.

[17]Henze, N. 2001. Open Adaptive Hypermedia: An approach to adaptive information presentation on the Web. Proceedings of the First International Conference on Universal Access in Human-Computer Interaction (UAHCI 2001), held jointly with HCI Internationa1, New Orleans, Louisiana, USA.

[18] International Organisation for Standardisation, ISO 9241-11: Ergonomic requirements for office work with visual display terminals (VDTs) - Part 11: Guidance on usability, International Organisation for Standardisation, Switzerland, 1998.

[19] Joachims, T., Freitag, D. and Mitchell, T. 1997. WebWatcher: a tour guide for the world wide web. Proceedings of the 15th International Joint Conference on Artificial Intelligence IJCAI97, Nagoya, Japan.

[20] Maneewatthana, T., Wills, G. and Hall, W. 2005. Adaptive Personal Information Environment based on the Semantic Web. Proceedings of HT 2005, Salzburg, Austria.

[21] Mladenic, D. 1996. Personal WebWatcher: Implementation and Design. Technical Report IJS-DP7472, Department of Intelligent Systems, J. Stefan Institute, Slovenia.

[22] Millard, D.E., Moreau, L., Davis, H.C. and Reich, S. 2000. FOHM: A Fundamental Open Hypertext Model for Investigating Interoperability between Hypertext Domains. Proceedings of the Eleventh ACM Conference on Hypertext and Hypermedia HT'00, San-Antonio, Texas, pp. 93-102.

[23] Millard, D.E., Davis, H.C., Weal, M.J., Aben, K. and De Bra, P. 2003. AHA! Meets Auld Linky: Integrating Designed and Freeform Hypertext Systems. Proceedings of ACM Hypertext'03, Nottingham, UK, pp. 161-169.

[24] Michaelides, D.T., Millard, D.E., Weal, M.J. and De Roure, D.C. 2001. Auld Leaky: A Contextual Open Hypermedia Link Server. Proceedings of the 7th Workshop on Open Hypermedia Systems, ACM Hypertext 2001 Conference, Denmark.

[25] Nielsen, J. 1993. Usability Engineering. Boston, Academic Press.

[26]Pearl, A. 1989. Sun's Link Service: A Protocol for Open Linking. Proceedings of the second annual ACM conference on Hypertext, 1989, Pittsburgh PA, pp. 137-146.

[27] Tsandilas, T. and schraefel, m.c. 2004. Usable Adaptive Hypermedia Systems. New Review of Hypermedia and Multimedia, 10(1), pp. 5-29. 
[28] Yankelovich, N., Haan, B.J., Meyrowitz, N.K. and Drucker, S.M. 1998. Intermedia: The Concept and the Construction of a Seamless Information Environment. Computer, 21(1), pp. 81-96.

[29]Fountain, A., Hall, W., Health, L. and Davis, H.C. 1990. Microcosm: an Open Model with Dynamic Linking. Proceedings of the European Conference on Hypertext (ECHT’90), pp 298-311.

[30]Collins, J. 2003. An Assessment of Multi-Dimensional Databases and Their Use [online]. Available from: http://www.designireland.net/alpha/controller/view_article.php?oid=00000000034.

[31]Babiker, E.M., Fujihara, H. and Boyle, C.D.B. 1991. A Metric for Hypertext Usability. Proceedings of the 9th Annual International Conference on Systems Documentation, ACM Press, USA, pp. 95-104.

[32] Crowder, R., Wills, G. and Hall, W. 2003. Evaluation of a hypermedia maintenance support application. Computers in Industry, 51 (3), pp. 327-344.

[33]De Bra, P., Brusilovsky, P. and Houben, G. 1999. Adaptive Hypermedia: From Systems to Framework. ACM Computing Surveys, 31(4es), Article 12.

[34] Halasz, F. and Schwartz, M. 1994. The Dexter hypertext reference model. Communications of the ACM, 37(2), pp. 30-39.

[35]Höök, K. 1997. Evaluating the Utility and Usability of an Adaptive Hypermedia System. Proceedings of 1997 International Conference on Intelligent User Interfaces, Orlando, FL, ACM, pp. 179-186.

[36] Hothi, J. 2001. Using an Open Hypermedia System to Develop New Techniques in Adaptive Hypermedia. PhD Thesis. School of Electronics and Computer Science, University of Southampton, UK.

[37]Kirakowski, J. and Corbett, M. 1993. SUMI: The Software Usability Measurement Inventory. British Journal of Educational Technology, Vol. 24, pp. 210-212.

[38]Leggett, J. and Schnase, J. 1994. Dexter with Open Eyes. Communications of the ACM, 37 (2), pp. 86-72.

[39] Newman, W. and Lamning, M. 1995. Interactive System Design. Addison Wesley.

[40] Nielsen, J. 1990. The art of navigating through hypertext. Communications of the ACM, 33, pp. 296-310.

[41] Nielsen, J. 1990. Evaluating Hypertext Usability. In: D.H. Jonassen, and H. Mandl, eds. Design Hypermedia for Learning. NATO ASI Series, Vol. 67, pp. 147-168, SpringerVerlag, Berlin.

[42] Weinreich, H. Obendorf, H. and Lamersdorf, W. 2001. The Look of the Link - Concepts for the User Interface of Extended Hyperlinks. Proceedings of the Twelfth ACM Conference on Hypertext and Hypermedia, Århus, Denmark, pp.19-28.

[43] Wright, P. 1991. Cognitive Overheads and Prostheses: Some Issues in Evaluating Hypertext. Proceedings of the 3rd ACM Conference on Hypertext, San Antonio, pp. 112.

[44]Kaj Gronbaek (Guest Editor) 1999 Open Hypermedia Systems. New Review of Hypermedia and Multimedia Vol 5 


\section{Appendix A Instructions given to the Expert Reviewers.}

\section{Heuristic Evaluation}

\section{Introduction}

The separation of links from documents (the Open Hypermedia fundamental) enables links to be created, added, or modified without any effect on the original document, and that despite the documents being modified or moved around the links would still function (Bailey et al., 2001).

Some problems with traditional links augmentation technique (a technique whereby links are inserted directly into the document) are that

- Every keyword can become a link, or there are too many links inserted into the document ('Prolific linking', Carr et al., 2002).

- Situations when links fail to present in the right document's context ('out of context' or irrelevant links') (El-Beltagy et al., 2002).

Adaptive Hypermedia techniques allow information (contents and links) to be personalised and adapted.

However, one of the main criticisms of the adaptive systems is that users are prevented from seeing the system's behaviour (Tsandilas and schraefel, 2004), and users have no control over the presentation of contents and links.

\section{Research Objectives}

- To provide a new application of the link augmentation technique by

- $\quad$ presenting a different view of representing a linkbase.

- $\quad$ solving some of the problems caused by the traditional open hypermedia technique ('Link Augmentation') that too many links are dynamically inserted into the document (prolific linking and irrelevant links).

- To deal with one of the criticisms of adaptive hypermedia that users are not allowed to have control over the personalisation and adaptation.

\section{The MDL Concept}

In a domain where there are many categories of users such as novices, beginners, or advanced users (and some stages in between) within a given context, or when there are many expertise dimensions required in the subject domain, the MDL concept can be beneficial. For instance, a user who is a skilled English historian but has no expertise in Asian history needs a different set of links presentation from a user who might be an Asian historian but has limited knowledge about English history.

The MDL concept is a notion that describes a single linkbase that contains links annotated with metadata. These different sets of links in the linkbase are treated as different dimensions of expertise and are encoded to condition the visibility of links and are dynamically inserted into the webpage when selected. If no links are chosen, the user will only see the common (static) structural links to navigate between web pages.

So, for instance, one link could be annotated as a member of the expert group while another in the same linkbase could be annotated as a member of novice group. At the same time users are provided with control over the presentation and personalisation of links. 


\section{Objectives of the Reviews}

To report any strengths and weaknesses and the applicability of the user-controlled adaptation provided by the MDL concept, as a possible additional functionality to solve some of the problems with links overloads and to allow the user control over the links presentation and personalisation.

The concept of MDL was applied and implemented in the development of the prototype system, called Inquiry-led Personalised Navigation System (IPNS).

There are three tools which allow the integration of the MDL concept and links presentation and personalisation.

- Tool 1: Personalised Links Assistant interface - this tool allows users to select the links to be displayed, based on their background and preference.

- Based on the concept that users have different levels of expertise and background, hence they should not have to see the links that they don't want to see.

- Another example, some users do not want to see all the links that they already know the meanings of, and they only want to see the links that correspond to their expertise levels, i.e. basic or beginner, advanced, etc.

- This concept also allows one given keyword to become links for an individual person, but not for another person, or some same keyword can become a link pointing to different destination based on the skill and preference of users. For instance, a keyword 'wheat', when users select Language as English, 'wheat' will be a link to its description. However, if the user selects Language as Latin, this same word 'wheat' will point to the its name in Latin.

- Tool 2: Inquiry Links - used to search for Concepts in the subject domain. This tool uses the same principle as the search engine but it is NOT a search engine. It will only display results when the searching word is in our concept relationships or domain ontology.

- Tool 3: Follow links - used to search for a word we want to know if there are any links related from this searched word. If there are, then it will know the links. Users need to highlight the word they are looking for and click the Select Text button at the end of each web page. 